\title{
Clinical spectrum and therapeutic approach to hepatocellular injury in patients with hyperthyroidism
}

This article was published in the following Dove Press journal:

Clinical and Experimental Gastroenterology

18 February 2013

Number of times this article has been viewed

\author{
Daniel Ferraz de Campos \\ Mazo' \\ Graciana Bandeira Salgado \\ de Vasconcelos' \\ Maria Adelaide Albergaria \\ Pereira ${ }^{2}$ \\ Evandro Sobroza de Mello 3 \\ Telesforo Bacchella' \\ Flair Jose Carrilho' \\ Eduardo Luiz Rachid \\ Cançado ${ }^{1,4}$ \\ 'Department of Gastroenterology, \\ ${ }^{2}$ Department of Internal Medicine, \\ ${ }^{3}$ Department of Pathology, ${ }^{4}$ Laboratory \\ of Immunopathology of Schistosomiasis \\ (LIM 06), Institute of Tropical Medicine, \\ University of São Paulo School of \\ Medicine, São Paulo, Brazil
}

Correspondence: Eduardo Luiz Rachid Cançado

Department of Gastroenterology, University of São Paulo School of

Medicine, Av Dr Eneas de Carvalho

Aguiar 255, $9^{\circ}$ andar, sala 9159 ,

São Paulo 05403-000, Brazil

Tel +55 | | 266 | 6447

Fax +55 II 266 I 7830

Email edulrc@uol.com.br

\begin{abstract}
Liver dysfunction in patients with hyperthyroidism includes abnormalities associated with the effects of thyroid hormone excess, those secondary to drug-induced liver injury, and changes resulting from concomitant liver disease. Our goal was to describe clinical, biochemical, and histopathological patterns in patients suffering from hyperthyroidism and concomitant liver dysfunction and to propose an algorithm of procedures to facilitate diagnosis and management of such cases. This study describes seven patients with liver biochemistry abnormalities detected after diagnosis of hyperthyroidism and one with undiagnosed decompensated hyperthyroidism and acute hepatitis. Two patients showed autoantibody reactivity which, together with liver histology, suggested the diagnosis of classic autoimmune hepatitis. Three patients experienced hepatotoxicity induced by propylthiouracil, the manifestations of which ranged from a benign course after drug withdrawal in one, a longstanding course in another suggesting drug-induced autoimmune hepatitis, and a more severe clinical condition with acute liver failure in a third patient, requiring liver transplantation. The three remaining patients showed no precipitating factors other than thyroid hyperactivity itself. They could be interpreted as having a thyroid storm with different clinical presentations. In conclusion, this series of patients illustrates the most frequent patterns of hepatocellular damage associated with hyperthyroidism and provides an algorithm for their diagnosis and treatment.
\end{abstract}

Keywords: thyroid disease, liver function tests, autoimmune hepatitis, drug-induced hepatitis, thyrotoxicosis hepatitis

\section{Introduction}

Liver dysfunction related to hyperthyroidism encompasses abnormalities associated with the effects of thyroid hormone excess, drug-related hepatic injury, and the presence of concomitant liver disease. ${ }^{1}$ In patients with hyperthyroidism who have never been treated, alterations in liver biochemistry are frequent, occurring in $45 \%-90 \%$ of this population ${ }^{2,3}$ and being usually mild and asymptomatic. ${ }^{4,5}$ Increases in serum alkaline phosphatase, often attributed to its bone fraction, ${ }^{6}$ followed in frequency by increases in levels of aminotransferases and bilirubin are also observed. Although less frequent, symptomatic cholestasis ${ }^{7}$ and hepatitis ${ }^{8}$ of variable intensity may also occur. When symptomatic hepatitis develops, a more severe disease known as thyrotoxic hepatitis ${ }^{9,10}$ occurs, but biochemical normalization is usually achieved after euthyroid status is reached. The aim of this paper was to report cases of hyperthyroidism-related liver dysfunction and to discuss the clinical spectrum of hepatocellular injury in this context along with the therapeutic approach.

Seven patients with a previous diagnosis of hyperthyroidism, including three with definite Graves' disease, who developed alterations in hepatic biochemistry after 
diagnosis of this disease, are included. A case of previously undiagnosed decompensated hyperthyroidism presenting as acute hepatitis is also described. Laboratory findings are shown in Table 1 and the main histological alterations on liver biopsy (performed in six of eight patients, and two at autopsy) in Table 2.

Aspartate aminotransferase, alanine aminotransferase, gamma glutamyl transpeptidase, and alkaline phosphatase were determined by a kinetic automatized method and bilirubin by a colorimetric automatized method. Thyroid hormones were determined by electrochemiluminometric assay. Antinuclear and antiactin antibodies were tested by indirect immunofluorescence in acetone-fixed HEp-2 cells and human fibroblasts, respectively. Antismooth muscle, antiliver kidney microsome type 1 , and anticollecting duct cell antibodies were detected by indirect immunofluorescence in unfixed murine tissue sections. Serum titers of 1:40 or higher were considered positive for antinuclear, antismooth muscle, and antiliver kidney microsome type 1, and from 1:10 for antiactin antibodies. Other laboratory tests were performed according to the standard techniques used in the laboratory at our institution.

The histological liver specimens were fixed in $4 \%$ formaldehyde and subjected to hematoxylin-eosin, Masson, and Picrosirius staining. Liver tissue examinations were performed by an experienced liver pathologist.

All patients were female and aged 15-43 years. The maximum interval between diagnosis of hyperthyroidism and clinical manifestation of liver disease was 4 years.
Five patients were using specific antithyroid therapy, four were taking propylthiouracil 200-900 (mean 325) mg/day, and one was taking methimazole $60 \mathrm{mg} /$ day. Three patients were not taking antithyroid drugs when the symptoms related to liver disease started. Independent of the causative factor, all patients had similar clinical manifestations, including nausea, vomiting, jaundice, dark urine, pale stools, abdominal pain, and diarrhea. None had symptoms or signs of hypersensitivity such as rash or urticaria. Serological markers for viral hepatitis were negative in all cases, as well as other specific markers of liver disease, except for antismooth muscle and antiliver kidney microsome type 1 antibodies in cases 3 and 4, respectively.

One patient showed significant recovery after propylthiouracil withdrawal and another, who had not been treated previously, became asymptomatic and had biochemical improvement after starting an antithyroid drug. Patients who had been receiving antithyroid drugs before the onset of liver symptoms had their therapeutic protocol changed, even if the medication in use was not the most probable cause of their liver disorder. Three patients with a favorable outcome received radioactive iodine as definite therapy after clinical compensation and two were controlled with antithyroid drugs. One patient underwent liver transplantation and two patients had a fatal outcome.

\section{Case I}

Case 1 was a 27-year-old woman who had taken propylthiouracil $600 \mathrm{mg} /$ day for 6 months because of Graves' disease.

Table I Exam results at onset of liver symptoms

\begin{tabular}{|c|c|c|c|c|c|c|c|c|}
\hline Biochemical findings & Case I & Case 2 & Case 3 & Case 4 & Case 5 & Case 6 & Case 7 & Case 8 \\
\hline AST $(x N V)$ & 37 & 2.17 & 13.0 & 80.3 & 3.4 & 3.2 & 20.2 & 35.7 \\
\hline ALT (xNV) & 34 & 2.4 & 9.15 & 45 & 2.6 & 1.9 & 29.7 & 23.6 \\
\hline Alkaline phosphatase (xNV) & 3.3 & 1.65 & 2.0 & 3.3 & 2.1 & 2.7 & 1.57 & 2.5 \\
\hline GGT $(x N V)$ & NA & 4.4 & 1.3 & NA & 0.4 & 2.3 & 5.9 & 1.7 \\
\hline Total bilirubin (mg/dL) & 17.5 & 30.7 & 0.4 & 23.9 & 20.5 & 19.4 & 11.7 & 26.8 \\
\hline Direct bilirubin $(\mathrm{mg} / \mathrm{dL})$ & 13.9 & 24.1 & 0.1 & 18.1 & 18.1 & 16.3 & 7.9 & 21.6 \\
\hline Protrombin activity (\%)/INR & NA & $25 / \mathrm{NA}$ & $61 / 1.3$ & $43 / 2.1$ & $47 / 1.5$ & $60 / 1.3$ & $69 / 1.2$ & $50 / 1.6$ \\
\hline Serum albumin $(g / d L)$ & 3.0 & 2.48 & 3.62 & 3.97 & 3.57 & 3.1 & 3.1 & 3.5 \\
\hline Serum globulin $(g / d L)$ & NA & 3.09 & 3.93 & 1.14 & 2.61 & NA & 1.70 & 2.7 \\
\hline Hemoglobin (g/dL) & 14.8 & 9.0 & II.I & 15.1 & 10.5 & 12.2 & 12.9 & 15.6 \\
\hline Hematocrit (\%) & 42.1 & 28.4 & 31.7 & 45.0 & 30.1 & 36.2 & 37.0 & 41.4 \\
\hline Leukocytes & 9200 & 5300 & 4970 & 5500 & 7100 & 4400 & 5300 & 7280 \\
\hline Platelets & 350,000 & 81,000 & 92,000 & 293,000 & 157,000 & 277,000 & 134,000 & 443,000 \\
\hline T3 (70-200 ng/dL) & 423 & 442 & 43 & 461 & 578 & 426 & 581 & 673 \\
\hline $\mathrm{T} 4(4.5-12 \mathrm{mcg} / \mathrm{dL})$ & $>24$ & $>20$ & 7.0 & 24.6 & 21 & $>24.9$ & NA & 22.4 \\
\hline Free T4 (0.6-I.54 ng/dL) & 3.1 & NA & 1.0 & $>6.4$ & $>6.2$ & NA & 7.1 & $>5.8$ \\
\hline $\mathrm{TSH}(0.5-4.2 \mathrm{mU} / \mathrm{mL})$ & 0.006 & 0.38 & 2.88 & $<0.03$ & $<0.03$ & $<0.005$ & $<0.01$ & $<0.03$ \\
\hline
\end{tabular}

Abbreviations: xNV, times normal value; NA, not available; AST, aspartate aminotransferase; ALT, alanine aminotransferase; GGT, gamma glutamyl transpeptidase; INR, international normalized ratio; TSH, thyroid-stimulating hormone; T3, triiodothyronine; T4, thyroxine. 
Table 2 Histological findings of liver biopsies

\begin{tabular}{|c|c|}
\hline Cases & Histology \\
\hline Case $2^{\dagger}$ & $\begin{array}{l}\text { Lobular structure is partially altered by thick septa containing } \\
\text { a moderate mixed inflammatory infiltrate; presence of } \\
\text { rosetting liver cells, hepatocyte ballooning, and an important } \\
\text { regenerative activity. Some portal spaces were mildly expanded } \\
\text { by edema and ductal proliferation. There were no pigments. }\end{array}$ \\
\hline Case 3 & $\begin{array}{l}\text { Irregular cirrhosis with severe periseptal activity; presence of } \\
\text { rosetting liver and plasma cells. }\end{array}$ \\
\hline Case $4^{\dagger}$ & $\begin{array}{l}\text { Chronic hepatitis with intense periportal activity with septa, } \\
\text { presence of numerous rosetting liver and plasma cells. }\end{array}$ \\
\hline Case 5 & Mild sinusoidal dilation, with bile plugs in biliary canaliculi. \\
\hline Case 6 & Presence of hemosiderin deposition and of mild sinusoidal dilation. \\
\hline Case 8 & $\begin{array}{l}\text { Severe acute hepatitis with zonal and panacinar necrosis } \\
\text { areas. }\end{array}$ \\
\hline
\end{tabular}

Note: tFirst biopsy result.

On admission to hospital, she had reported jaundice, pale stools, dark urine, weakness, nausea, vomiting, diffuse abdominal pain, and itching during the previous 15 days. Physical examination revealed intense jaundice, bilateral exophthalmos, an enlarged and painless thyroid, no murmurs, and diffuse abdominal pain, especially in the right and left upper quadrants, without organomegaly. Propylthiouracil was discontinued and aminotransferases returned to baseline levels. Radioactive iodine was administered as the definite treatment for hyperthyroidism. This case was considered a typical example of drug-induced liver disease. ${ }^{11}$ Although symptoms appeared only 180 days after introduction of propylthiouracil, the results of laboratory tests were normal after its discontinuation.

\section{Case 2}

Case 2 was a 24-year-old woman with known hyperthyroidism who had been taking propylthiouracil $600 \mathrm{mg}$ /day for the previous 6 months. She presented at first with complaints of nausea, vomiting, jaundice, abdominal enlargement, and dark urine. Although propylthiouracil was withdrawn, she became mentally confused, so was arbitrarily treated with corticosteroids because of a probable diagnosis of autoimmune hepatitis, and with therapeutic measures for hepatic encephalopathy. She improved steadily and was discharged on prednisone $15 \mathrm{mg} /$ day. Four months later, she presented to our institution with fever and painful arthropathy. On examination, mild jaundice, hepatomegaly, violaceous abdominal streaks, and painful joints (ankles, knees, left coxofemoral joint, shoulders, and lumbar region) were observed. At that time, her liver biochemistry showed only mild changes. Coxofemoral pyoarthritis was diagnosed and required surgical drainage. Prednisone was slowly tapered off and withdrawn. The patient underwent two liver biopsies at 2 and 9 months after discharge from hospital, both showing chronic moderately active hepatitis and septa with no etiologic markers. Although of questionable benefit, prednisone and azathioprine were administered and continued for 6 months until the patient became pregnant. She remained asymptomatic off medication, with normal biochemistry. She had a partial thyroidectomy 2 years after onset of symptoms, and underwent a third liver biopsy 2 years after the second one, and no inflammatory activity was observed.

Despite the long period of time required for histology to become normal, we assume that the etiology was the same as in the previous case, ie, drug-induced liver disease. Autoimmune hepatitis was also a strong diagnostic possibility, given that liver biopsies showed moderate inflammatory activity and the patient had a history of treatment with propylthiouracil, which is a known trigger of autoimmune liver damage. ${ }^{12-14}$ These considerations led us to opt for immunosuppressive therapy. Prednisone and azathioprine were given for 6 months only and there was complete histological recovery, with no signs of recurrence 20 months after discontinuing immunosuppressive drugs. This clinical course favors the diagnosis of drug-induced hepatitis or drug-induced autoimmune hepatitis, given that a benign course of autoimmune hepatitis without relapse after 6 months of treatment in a young female is very unusual indeed. ${ }^{15}$ The severity of liver injury perhaps accounted for the delayed biochemical and complete histological recovery in this case.

\section{Case 3}

Case 3 was a 21-year-old woman who had had hyperthyroidism for one year and without specific treatment. Ten months prior to hospital admission, she had complained of tachycardia, myalgia, jaundice, and dark urine. Thyroid crisis was diagnosed, and propylthiouracil was prescribed together with oral iodine and corticosteroids. She developed thrombocytopenia, with no changes in myelogram, but the prednisone dose was increased to $1 \mathrm{mg} / \mathrm{kg} /$ day following advice from a hematologist. On that occasion, she had had elevated hepatic enzymes, initially interpreted as secondary to thyroid crisis, which decreased after corticosteroid therapy. Upon recovery, she was discharged on the medications already mentioned. Within 30 days, she returned to the emergency room with decompensated diabetes. The corticosteroid dose was reduced and diabetes mellitus was treated initially with insulin. Thereafter, the aminotransferase values again increased progressively. Propylthiouracil was replaced by methimazole, based on the hypothesis that 
the patient had developed drug-induced hepatotoxicity. She was followed up as an outpatient and showed progressive elevation of hepatic enzymes, although thyroid tests had reverted to normal. Antismooth muscle antibodies with glomerular pattern were reactive up to $1 / 80$, with specificity for microfilament antigens (antiactin antibodies). Liver biopsy showed irregular cirrhosis with very intense interface and lobular activity, numerous plasma cells, and rosetting of hepatocytes (Figure 1). After introduction of prednisone and azathioprine, aminotransferase values reverted to the normal range in the following months. This patient had the widest differential diagnosis at clinical presentation. The aminotransferases were increased even before specific therapy for hyperthyroidism was started, leading to an initial diagnosis of liver damage secondary to thyroid hyperactivity. Although liver enzymes improved when antithyroid drugs were initiated, prednisone, which was prescribed for thrombocytopenia, could have been involved in lowering of her aminotransferase levels. After suspension of corticosteroid therapy, the aminotransferase levels increased again, reaching even higher values than before. Finally, the presence of autoantibody reactivity along with liver biopsy findings led to the diagnosis of autoimmune hepatitis type $1 .{ }^{15}$

\section{Case 4}

Case 4 was a 15 -year-old girl who had been diagnosed with Graves' disease 4 years prior to admission. She had been treated irregularly with propranolol $40 \mathrm{mg} /$ day and propylthiouracil $300 \mathrm{mg} /$ day. She described dark urine, acholic stools, vomiting, and malaise in the previous 45 days.

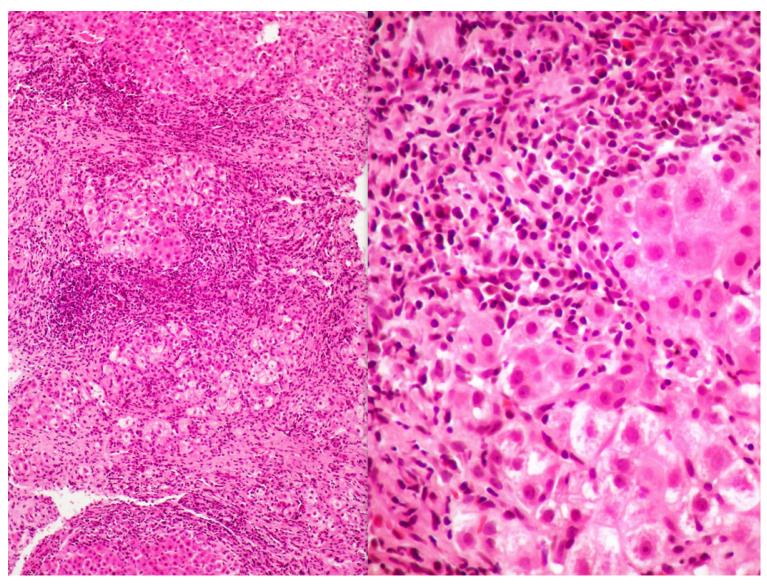

Figure I Case 3, showing cirrhosis with large septa and intense inflammation, a prominent interface, lobular activity, and hepatocyte rosetting (left, hematoxylin and eosin, 100x).

Notes: At higher magnification, the numerous plasma cells and rosetting of hepatocytes are very conspicuous, in addition to the interface hepatitis (right, hematoxylin and eosin, 400×).
She was admitted in a poor state of health, with dehydration, jaundice, emaciation, bilateral exophthalmos, and thyroid enlargement. Propylthiouracil was suspended and treatment with hydrocortisone $150 \mathrm{mg}$ /day, oral iodine $1.5 \mathrm{~g} /$ day, and methimazole $60 \mathrm{mg}$ /day was initiated, because of diagnosis of a thyroid storm. One month later, the liver enzymes decreased to $50 \%$ of the levels found on admission, but the patient developed ascites and hepatic encephalopathy, which responded favorably to specific medical treatment and empirical antibiotic therapy. Severe hypokalemia, metabolic acidosis, and reactivity of anticollecting duct cell antibodies $(>1 / 320)$ led to the diagnosis of distal renal tubular acidosis. After clinical improvement, the patient underwent a liver biopsy. Histological findings (Table 2) together with persistence of hepatic biochemical abnormalities and reactivity of antiliver kidney microsome type 1 antibodies led to a diagnosis of type 2 autoimmune hepatitis. Prednisone and azathioprine were prescribed based on this diagnosis.

Two months later, the patient received radioactive iodine for thyroid ablation. After 6 months, the liver enzyme levels returned to the normal range and she remained asymptomatic. Three liver biopsies were performed after biochemical remission, with a mean interval of 18 months between each biopsy, until histological remission was reached. The patient remained without clinical and laboratory signs of recurrence and was on no medication. Antibodies against collecting duct cells have been described in autoimmune disease of variable etiology, such as thyroid disease, pernicious anemia, and autoimmune hepatitis, especially type 2 in our experience. ${ }^{16}$

\section{Case 5}

Case 5 was a 26-year-old woman diagnosed with hyperthyroidism 18 months before admission, who had been on treatment with methimazole $60 \mathrm{mg}$ /day and propranolol $40 \mathrm{mg} /$ day for 12 months. In the previous 3 months, she had developed worsening jaundice, pain in the right upper abdominal quadrant, and vomiting, with suspension of both drugs in the previous 30 days. She also complained of dyspnea, lower limb edema, and oliguria, which worsened after withdrawal of methimazole. Clinical evaluation revealed moderate jaundice, mild dehydration, right lower lung crackles, painful hepatomegaly, mild peripheral edema, and tremors of the extremities. She had atrial fibrillation with a high ventricular rate, and a chest X-ray showed pleural effusion. A diagnosis of thyroid storm and right-sided heart failure was made, and she received oral iodine, captopril, digitalis, and diuretics. Despite therapy, her liver function and general status worsened. In an attempt to compensate for her hypercatabolic state, methimazole 
$90 \mathrm{mg}$ /day was reintroduced and prednisone $20 \mathrm{mg} /$ day was started. About 20 days after hospital admission, she developed a urinary tract infection and progressed to liver failure. Major complications, including coagulopathy, hepatic encephalopathy, and gastrointestinal bleeding occurred, without response to medical therapy. Hepatic tissue samples obtained during autopsy revealed mild sinusoidal dilation with bile plugs in the bile canaliculi. Other considerations about this case are addressed along with case 6 .

\section{Case 6}

Case 6 was a 26-year-old woman who had been receiving propylthiouracil $200 \mathrm{mg}$ /day for hyperthyroidism over the previous 3 years. Two months before hospital admission, she developed anorexia, weight loss, pruritus, dark urine, abdominal distension, and watery diarrhea, with fever in the previous few days. On physical examination, she was agitated, pale, moderately jaundiced, tachycardic, and had a systolic murmur $(2+/ 6+)$ at the left lower sternal border. Pulmonary auscultation and abdominal examination were normal and there was no edema of the lower extremities. Again, decompensated hyperthyroidism was diagnosed, and oral iodine $1 \mathrm{~g}$ /day, propranolol $80 \mathrm{mg}$ /day, and hydrocortisone $300 \mathrm{mg}$ /day were initially prescribed. Antibiotics (ceftriaxone and clindamycin) were introduced empirically, despite a negative screen for infection. The propylthiouracil dose was increased to $800 \mathrm{mg} /$ day in an attempt to control the thyrotoxicosis, but later had to be replaced by lithium carbonate because of leucopenia. Fever persisted without any apparent focus of infection, and a change in antibiotic (ceftazidime and later a combination of imipenem, vancomycin, and amphotericin) did not result in clinical improvement. She then became agitated and was transferred to the intensive care unit. Neurological imaging and liquor were normal. Her level of consciousness steadily deteriorated in the ensuing days, and she developed status epilepticus and signs of congestive heart failure associated with fatal multiple organ dysfunction. Autopsy revealed marked liver congestion secondary to heart failure and well defined areas of centrilobular necrosis (Figure 2).

Unlike the first four patients, no trigger for liver injury was found in cases 5 and 6 other than thyroid hyperactivity. Thyroid storm is an extreme form of thyrotoxicosis, characterized by fever, delirium, tachycardia, hypotension, vomiting, jaundice, and diarrhea. ${ }^{17}$ Heart failure may have contributed to the higher morbidity and subsequent death as a result of probable thyroid storm in cases 5 and 6 . These patients showed a mild increase in aminotransferase levels,

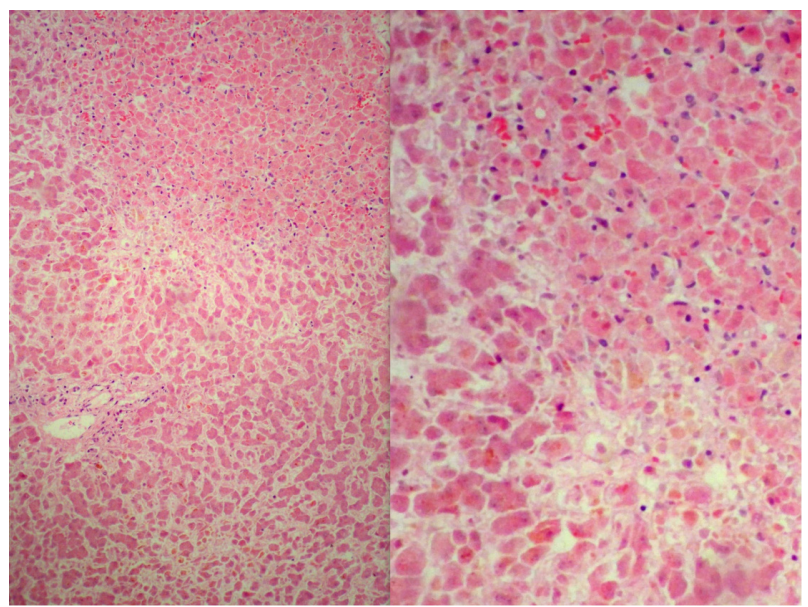

Figure 2 Case 6, with microscopic findings in the liver at autopsy.

Notes: There is marked sinusoidal congestion and centrilobular necrosis (left, hematoxylin and eosin, $100 \times$ ). Centrilobular necrosis is shown in detail at right (hematoxylin and eosin, 400x).

but a significant increase in bilirubin when compared with the other six patients who did not have heart failure, and high levels of bilirubin have previously been described as a marker of disease severity. ${ }^{18}$

\section{Case 7}

Case 7 was a 43-year-old woman who was admitted to another hospital complaining of upper abdominal pain and diarrhea for 2 weeks and jaundice for the previous 24 hours. She denied other disease, but when asked, reported 10 months of weight loss, agitation, tremor, and hair loss. A medical consultation had been sought 5 months earlier because of these complaints, and a diagnosis of panic disorder was made. For one month, she had used a drug combination of fluoxetine, propranolol, and clonazepam, which she then stopped without taking medical advice. At admission, she had jaundice and a tender liver edge palpable $1.5 \mathrm{~cm}$ below the right costal margin. There was no exophthalmos or thyroid enlargement. Liver tests showed mainly elevated serum aminotransferases and progressive hyperbilirubinemia. There was no biliary disease on abdominal magnetic resonance imaging, but hepatomegaly and perihepatic ascites were detected. Because early tests were negative for viral hepatitis and progressive hyperbilirubinemia developed, prednisone $20 \mathrm{mg}$ were prescribed on the suspicion of autoimmune hepatitis, pending further laboratory evaluation. Hyperthyroidism was finally confirmed, and propranolol $120 \mathrm{mg} /$ day, hydrocortisone $400 \mathrm{mg} /$ day, and lithium carbonate $900 \mathrm{mg}$ /day were given on the recommendation of an endocrinologist, and on which she became asymptomatic with normalization of liver enzymes. Corticosteroids were administered only during 
the first 10 days. She continues to be seen as an outpatient, receives methimazole and propranolol, and awaits ablative therapy for hyperthyroidism.

This patient had clinical and laboratory features of acute hepatitis. Her symptoms could have been masked by propranolol, prescribed for panic disorder, and probably delayed appropriate treatment for thyroid dysfunction. Improvement in her symptoms and hepatic biochemical parameters were seen when she became euthyroid, but the patient had received corticosteroids for ten days. Although less probable, concomitant autoimmune hepatitis was difficult to rule out during early follow-up. However, aminotransferase levels remained within the normal range even one year after discontinuation of corticosteroids, and this argues strongly in favor of liver injury secondary to hyperthyroidism.

\section{Case 8}

Case 8 was a 19-year-old woman with hyperthyroidism diagnosed 5 months earlier during a medical evaluation for tremors, weight loss, diarrhea, cervical enlargement, and eye protrusion. Propylthiouracil $900 \mathrm{mg} /$ day was prescribed, but the patient only started using it 2 months later and irregularly. Two months before admission, use of propylthiouracil was reinforced, in combination with propranolol $120 \mathrm{mg} /$ day. At admission to the emergency unit, she had jaundice, vomiting, diarrhea, and dark urine. Her clinical symptoms deteriorated rapidly, with development of hypoglycemia and mental depression. Due to worsening of liver parameters and considering the possibility of thyrotoxicosis in association with hepatotoxicity induced by propylthiouracil, she underwent plasmapheresis, followed by urgent thyroidectomy, and later was prioritized for liver transplantation. The liver explant showed extensive panacinar necrosis, typical of fulminant hepatitis (Figure 3 ). She remains well, attending an outpatient clinic for follow-up. In this case, the antithyroid drug and thyroid hormones could have been responsible for the clinical and biochemical liver abnormalities seen. ${ }^{19}$ The decision to perform an urgent ablation for hyperthyroidism was based on the potentially harmful effects that excessive thyroid hormones might exert on propylthiouracil-related hepatotoxicity. Plasmapheresis was effective in preparing the patient for emergency surgery, as reported in the medical literature. ${ }^{20}$ This case also shows that liver transplantation remains a feasible and effective treatment option for severe drug-induced liver dysfunction.

\section{Discussion}

The etiology of liver injury in patients with hyperthyroidism covers a broad diagnostic spectrum, and more than one cause

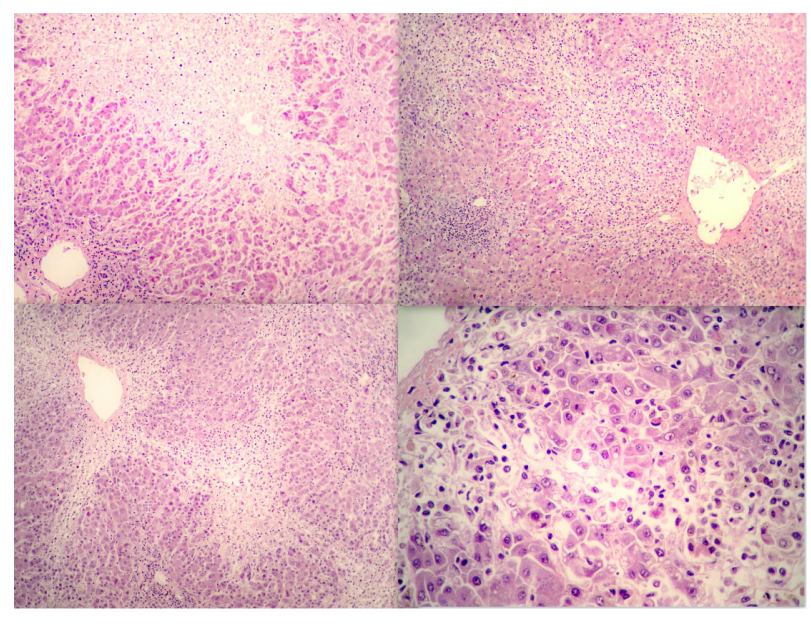

Figure 3 Case 8 , in whom extensive areas of panacinar necrosis with variable inflammation (top left and top right, hematoxylin and eosin, 100×) was the dominant picture.

Notes: In a few areas with more preserved parenchyma, there was bridge necrosis (bottom left, hematoxylin and eosin, 40x), and at higher magnification, numerous apoptotic bodies and mixed inflammation (bottom right, hematoxylin and eosin, $400 \times)$.

may be identified at presentation. Sometimes the diagnosis is possible only after a long period of monitoring. Occasionally, definitive diagnosis remains unclear even after a lengthy observation period.

These cases are reported in an attempt to illustrate the main types of hepatocellular disorder which occur in patients with hyperthyroidism, both before and during its treatment. These disorders may result from the effects of thyroid hormone excess, drug-induced liver injury, and sometimes pre-existing or concomitant liver disease.

Thyroid hormone excess causes an increase in cardiac output without an increase in hepatic blood flow ${ }^{21,22}$ which, in turn, leads to increased oxygen consumption in the liver. The combination of increased oxygen consumption and decreased perfusion leads to tissue hypoxia, contributing significantly to the liver dysfunction that occurs in patients with hyperthyroidism. ${ }^{2}$ Histological examination shows necrosis in zone 3 , an injury typically occurring secondary to a relative decrease in blood supply. ${ }^{23}$ Exacerbations of these hemodynamic changes may be seen during a thyrotoxic storm, ${ }^{17}$ when direct effects of excess thyroid hormone are combined with a higher oxygen requirement by mitochondrial-based metabolism within the hepatocytes. ${ }^{2,24}$

Right-sided heart failure associated with severe hyperthyroidism contributes to worsening of hepatic injury, ${ }^{2,25}$ and usually presents with mild alterations of liver function tests. However, in cases of acute congestion, aminotransferase levels may reach values as high as those associated with viral or toxic hepatitis, and bilirubin may exceed $20 \mathrm{mg} / \mathrm{dL}$, 
so may act as a marker of disease severity ${ }^{18}$ In this situation, histological examination of the liver shows changes ranging from dilation of the centrilobular veins and sinusoids to necrosis of hepatocytes in the centrilobular region.

Hepatotoxicity due to antithyroid drugs is very rare, is estimated to occur in less than $0.5 \%$ of users, ${ }^{26}$ and is much more frequent with propylthiouracil than with methimazole. ${ }^{27}$ Antithyroid drugs are metabolized mainly by the liver, with renal clearance of $80 \%$ for methimazole and $35 \%$ for propylthiouracil. ${ }^{28}$ The main alterations related to methimazole are those of cholestasis, while those associated with propylthiouracil are hepatocellular. ${ }^{29,30}$ Propylthiouracil most commonly causes a mild transient asymptomatic increase in aminotransferases ${ }^{29,31}$ and does not require drug withdrawal, but symptomatic hepatic injury has also been reported. Propylthiouracil hepatotoxicity usually appears during the first 3 months of therapy, probably triggered by an immunoallergic reaction which is as yet not fully understood. ${ }^{28,32}$ The prognosis is usually benign, but severe hepatic dysfunction may occur. There are some reports of autoimmune hepatitis associated with hyperthyroidism. ${ }^{33,34}$ Considering that both diseases are frequent in young women and can be associated with other autoimmune disorders, when symptoms of liver and thyroid disease appear together, the presence of both conditions should be suspected. There have been reports of autoantibody reactivity, clinical manifestations of vasculitis, ${ }^{12,13}$ and even hepatotoxicity in patients receiving propylthiouracil, sometimes mimicking autoimmune hepatitis. ${ }^{14}$ It is not clear if these findings represent a drug-mediated phenomenon or a spectrum of abnormal immune function in Graves' disease.

In this study, an algorithm (Figure 4) is suggested for diagnosis and management of such patients. When faced

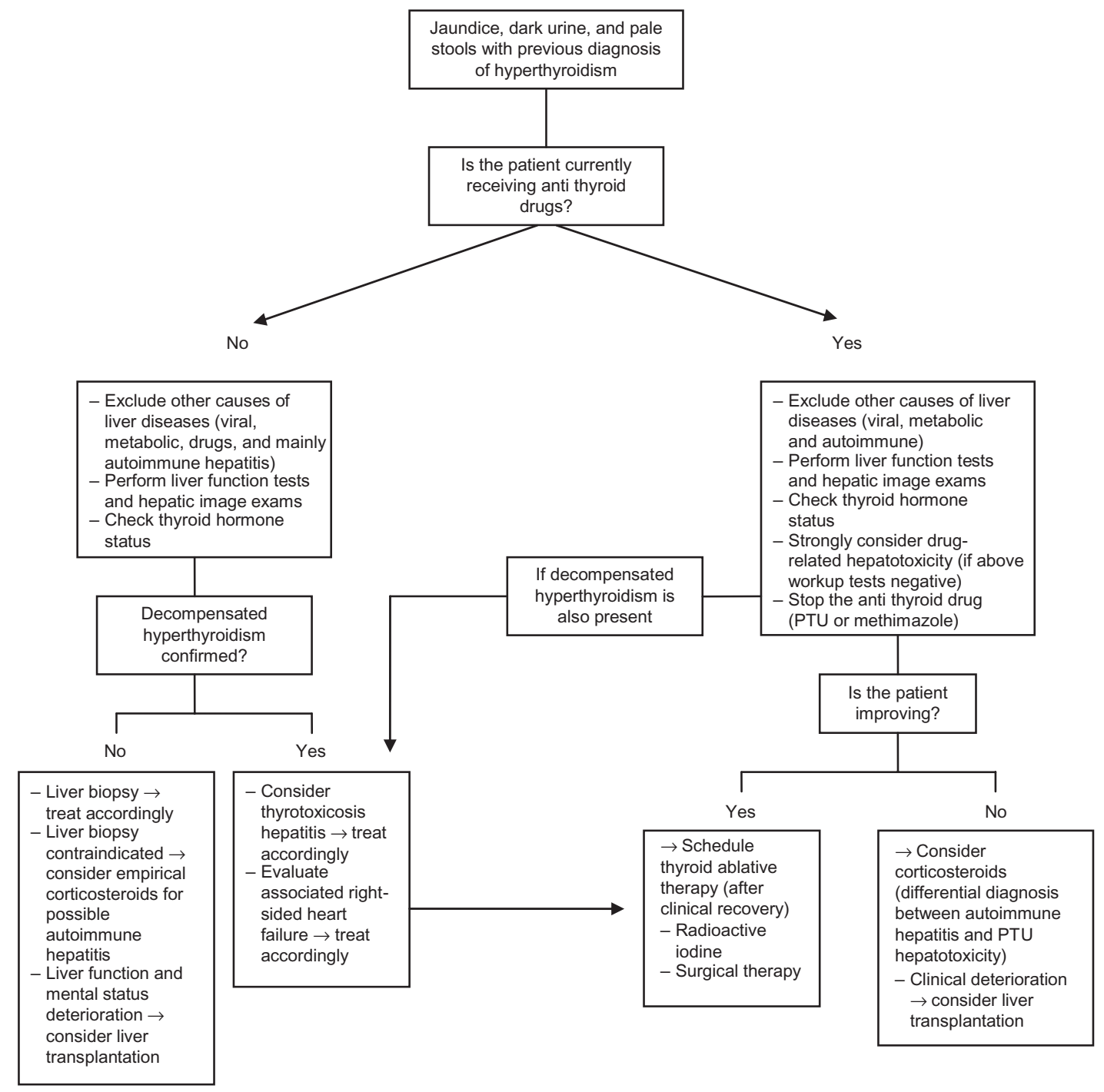

Figure 4 Algorithm for management of patients with symptoms of liver dysfunction and a previous diagnosis of hyperthyroidism. Abbreviation: PTU, propylthiouracil. 
with a patient reporting symptoms suggestive of liver disease and known hyperthyroidism, it is mandatory to check for use of antithyroid drugs. If the patient is on antithyroid therapy, and other types of liver disease have been excluded, suspicion should be raised for drug-induced hepatotoxicity and antithyroid medications should be suspended. It is important to remember that use of antithyroid treatment does not necessarily mean that hyperthyroidism is compensated, as described in some of the present cases. Therefore, it is important to check thyroid hormone levels.

When decompensated hyperthyroidism is present, thyrotoxic hepatitis alone or in association with drug-induced hepatotoxicity should be considered, whether or not there is right-sided heart failure. When a favorable clinical response occurs using the initial approach, such as drug interruption or compensation for hyperthyroidism, we recommend subsequent thyroid ablation. On the other hand, if there is no response to initial therapy, corticosteroids might be used empirically, considering the possibility of autoimmune hepatitis or propylthiouracil hepatotoxicity. Liver transplantation is recommended in the event of severe drug-induced liver dysfunction with progressive clinical deterioration.

If the patient is not on antithyroid drugs and other types of liver disease, particularly autoimmune hepatitis, can be excluded, thyroid hormone status should be checked. In a patient without decompensated hyperthyroidism at the time of evaluation, a liver biopsy should be considered to investigate for an unexplained liver disorder. If liver biopsy is not feasible or contraindicated, empirical corticosteroids for possible autoimmune hepatitis could be the next step. In the event of lack of improvement and even deterioration, hepatic transplantation should be considered.

In conclusion, this series of patients highlights the most frequent forms of hepatocellular injury in patients with hyperthyroidism whether they are receiving antithyroid therapy or not. Sometimes it may be difficult to define clearly the cause of the liver abnormalities found. Association with autoimmune hepatitis is common, especially if Graves' disease is present. On the other hand, propylthiouracil-induced liver injury is rare, but may be very severe, even leading to acute liver failure that requires liver transplantation. Sometimes the definitive diagnosis of propylthiouracil-induced or classic autoimmune hepatitis can only be obtained during clinical follow-up, depending on whether or not there is a complete response to prescribed treatment, and whether or not the disorder recurs after treatment withdrawal. Other contributors to liver damage, such as ischemic liver damage secondary to a hyperdynamic state and associated right-sided heart failure, should be kept in mind.

\section{Disclosure}

The authors report no conflict of interests in this work.

\section{References}

1. Khemichian S, Fong TL. Hepatic dysfunction in hyperthyroidism. Gastroenterol Hepatol (N Y). 2011;7:337-339.

2. Youssef WI, Mullen KD. The liver in other (nondiabetic) endocrine disorders. Clin Liver Dis. 2002;6:879-889.

3. Kubota S, Amino N, Matsumoto Y, et al. Serial changes in liver function tests in patients with thyrotoxicosis induced by Graves' disease and painless thyroiditis. Thyroid. 2008;18:283-287.

4. Babb RR. Associations between diseases of the thyroid and the liver. Am J Gastroenterol. 1984;79:421-423.

5. Fong TL, McHutchinson JG, Reynolds TB. Hyperthyroidism and hepatic dysfunction. A case series analysis. J Clin Gastroenterol. 1992;14:240-244.

6. Huang MJ, Li KL, Wei JS, Wu SS, Fan KD, Liaw YF. Sequential liver and bone biochemical changes in hyperthyroidism: prospective controlled follow-up study. Am J Gastroenterol. 1994;89:1071-1076.

7. Hull K, Horenstein R, Naglieri R, Munir K, Ghany M, Celi FS. Two cases of thyroid storm-associated cholestatic jaundice. Endocr Pract. 2007;13:476-480

8. Choudhary AM, Roberts I. Thyroid storm presenting with liver failure. J Clin Gastroenterol. 1999;29:318-321.

9. Bellassoued M, Mnif M, Kaffel N, et al. Thyrotoxicosis hepatitis: a case report. Ann Endocrinol (Paris). 2001;62:235-238.

10. Barzilay-Yoseph L, Shabun A, Shilo L, Hadary R, Nabriski D, Kitay-Cohen Y. Thyrotoxic hepatitis. Isr Med Assoc J. 2011;13: 448-450.

11. Bénichou C. Criteria of drug-induced liver disorders. Report of an international consensus meeting. J Hepatol. 1990;11:272-276.

12. Dolman KM, Gans RO, Vervaat TJ, et al. Vasculitis and antineutrophil cytoplasmic autoantibodies associated with propylthiouracil therapy. Lancet. 1993;342:651-652.

13. Vogt BA, Kim Y, Jennette JC, Falk RJ, Burke BA, Sinaiko A. Antineutrophil cytoplasmic autoantibody-positive crescentic glomerulonephritis as a complication of treatment with propylthiouracil in children. J Pediatr. 1994;124:986-988.

14. Maggiore G, Larizza D, Lorini R, De Giacomo C, Scotta MS, Severi F. Propylthiouracil hepatotoxicity mimicking autoimmune chronic active hepatitis in a girl. J Pediatr Gastroenterol Nutr. 1989;8:547-548.

15. Alvarez F, Berg PA, Bianchi FB, et al. International Autoimmune Hepatitis Group report: review of criteria for diagnosis of autoimmune hepatitis. J Hepatol. 1999;31:929-938.

16. Farias AQ. Acidose tubular renal distal nas doenças hepáticas: análise dos fatores de risco (tese). São Paulo, Brazil: Faculdade de Medicina; 2001. Portuguese.

17. Burch HB, Wartofsky L. Life-threatening thyrotoxicosis. Thyroid storm. Endocrinol Metab Clin North Am. 1993;22:263-277.

18. Kay PS, Keefe EB. Cardiac disease. In: Gitlin N, editor. The Liver and Systemic Diseases. New York, NY: Churchill Livingston; 1997.

19. Kandil E, Khalek MA, Thethi T, Abd Elmageed Z, Khan A, Jaffe BM. Thyroid storm in a patient with fulminant hepatic failure. Laryngoscope. 2011;121:164-166.

20. Ezer A, Caliskan K, Parlakgumus A, Belli S, Kozanoglu I, Yildirim S. Preoperative therapeutic plasma exchange in patients with thyrotoxicosis. J Clin Apher. 2009;24:111-114.

21. Myers JD, Brannon ES, Holland BC. A correlative study of the cardiac output and the hepatic circulation in hyperthyroidism. J Clin Invest. 1950;29:1069-1077.

22. Shimizu Y. Liver in systemic disease. World J Gastroenterol. 2008;14: 4111-4119. 
23. Jansen PL, Froeling PG, Schade RW, Kloppenborg PW, Yap SH, Van Haelst UJ. Intrahepatic cholestasis in hyperthyroidism and the effect of antithyroid and beta-blocking drugs. Neth J Med. 1982;25: 318-324.

24. Goglia F, Liverini G, Lanni A, Barletta A. Mitochondrial DNA, RNA and protein synthesis in normal, hypothyroid and mildly hyperthyroid rat liver during cold exposure. Mol Cell Endocrinol. 1988;55:141-147.

25. Carithers RL. Endocrinologic disease. In: Gitlin N, editor. The Liver and Systemic Diseases. New York, NY: Churchill Livingston; 1997.

26. Cooper DS. Treatment of thyrotoxicosis. In: Braverman LE, Utiger RD editors. Werner and Ingbar's The Thyroid, 7th ed. Philadelphia, PA: Lippincott-Raven; 2004.

27. Cooper DS. Antithyroid drugs. N Engl J Med. 2005;352:905-917.

28. Solá Izquierdo E, Morillas Ariño C, Peña Guillermo H, Muñoz Ferrer ML, Morillas Ariño J, Hernández Mijares A. Hepatitis colestásica por metimazol. Endocrinol Nutr. 2002;49:127-129. Spanish.

29. Liaw YF, Huang MJ, Fan KD, Li KL, Wu SS, Chen TJ. Hepatic injury during propylthiouracil therapy in patients with hyperthyroidism. A cohort study. Ann Intern Med. 1993;118:424-428.
30. Majeed M, Babu A. Cholestasis secondary to hyperthyroidism made worse by methimazole. Am J Med Sci. 2006;332:51-53.

31. Aydemir S, Bayraktaroglu T, Demircan N, et al. Effect of hyperthyroidism and propylthiouracil treatment on liver biochemical tests. Int J Clin Pract. 2005;59:1304-1308.

32. Casallo Blanco S, Valero MA, Marcos Sanchez F, de Matias Salces L, Blanco Gonzalez JJ, Martin Barranco MJ. Methimazole and propylthiouracil induced acute toxic hepatitis. Gastroenterol Hepatol. 2007;30: 268-270.

33. Nagai T, Imamura M, Kamiya Y, Mori M. Graves' disease accompanied by anti-myeloperoxidade antibody-related nephropathy and autoimmune hepatitis. Intern Med. 2004;43:516-520.

34. Nobili V, Liaskos C, Luigi G, Guidi R, Francalanci P, Marcellini M. Autoimmune thyroiditis associated with autoimmune hepatitis. Thyroid 2005;5:1193-1195.
Clinical and Experimental Gastroenterology

\section{Publish your work in this journal}

Clinical and Experimental Gastroenterology is an international, peerreviewed, open access journal, publishing all aspects of gastroenterology in the clinic and laboratory, including: Pathology, pathophysiology of gastrointestinal disease; Investigation and treatment of gastointestinal disease; Pharmacology of drugs used in the alimentary tract;

\section{Dovepress}

Immunology/genetics/genomics related to gastrointestinal disease. This journal is indexed on CAS. The manuscript management system is completely online and includes a very quick and fair peer-review system. Visit http://www.dovepress.com/testimonials.php to read real quotes from published authors.

Submit your manuscript here: http://www.dovepress.com/clinical-and-experimental-gastroenterology-journal 